\title{
3 Research Square

\section{Did More Generous Health Insurance Improve Health Outcomes of The Elderly? Evidence From China}

Jusheng Shieh

Nanjing University

Xinpeng Xu ( $\nabla$ xuxinpeng@njmu.edu.cn )

Nanjing Medical University

Hua You

Nanjing Medical University

Qifeng Wu

Nanjing Medical University

\section{Research Article}

Keywords: Catastrophic Medical Insurance, health outcomes, elderly, China

Posted Date: November 19th, 2021

DOI: https://doi.org/10.21203/rs.3.rs-1091084/v1

License: (c) (i) This work is licensed under a Creative Commons Attribution 4.0 International License. Read Full License 
1 Did more generous health insurance improve health outcomes of the elderly? 2 evidence from China.

3

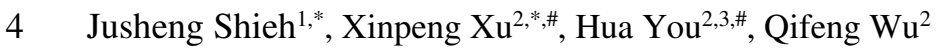

$5 \quad{ }^{1}$ Center for Health Policy and Management studies, Nanjing University, Nanjing, China

$6 \quad{ }^{2}$ School of Public Health, Nanjing Medical University, Nanjing, China

$7 \quad{ }^{3}$ Institute of Healthy Jiangsu Development, Nanjing Medical University, Nanjing, China

8

$9 \quad{ }^{\#}$ Corresponding Author: Xinpeng Xu, Hua You.

10 Address: School of Public Health, Nanjing Medical University, Nanjing, 211166, China

11 Email: xuxinpeng@njmu.edu.cn; youhua98@163.com

12

13 " Shieh, Jusheng and Xinpeng Xu contributed to this study equally.

14 
1 Did more generous health insurance improve health outcomes of the elderly? evidence from China.

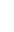

\section{Abstract}

Background: Catastrophic Medical Insurance (CMI) has been piloted in China Since 2012 and gradually implemented in various regions. Most studies focus on its impact on medical economic risks, and few studies discussed the impact of CMI on health of the elderly.

Methods: This study used Chinese Longitudinal Healthy Longevity Survey (CLHLS) data to explore the impact of CMI on health of the elderly. Difference-in-differences (DID) and Propensity score matching-DID were employed to study the health impact of CMI. Heckman selection model was used to study the potential mechanisms.

Results: We found that the implementation of CMI improved the mental health of the elderly, and the effect was limited. Moreover, the positive effect of CMI on the health of the elderly was mainly in the high-income group, and CMI had no significant effect on any health indicators of the lowincome group population. The potential channel of CMI health improvement was its ability to increase hospitalization rate in the elderly.

Conclusions: Therefore, the health promotion and equity of CMI deserve more attention, and the compensation level of CMI needs to be improved under the premise of sustainable and effective supervision of the fund.

Key words: Catastrophic Medical Insurance; health outcomes; elderly; China 


\section{Background}

(

The challenge of aging has prompted countries around the world to pay attention to aging health. According to China's seventh population census in 2020, people aged 60 and above accounted for $18.7 \%$ of the national population, an increase of $5.44 \%$ compared with the sixth population census[1]. For the elderly, the degeneration of physical function will increase the health vulnerability of this group because of the changes in physical and psychological aspects caused by the aging stage. According to the Healthy China Action (2019-2030), 75\% of the elderly in China suffered from one or more chronic diseases, and about 40 million of them are completely or partially disabled[2]. According to the survey data of China Scientific Research Center on Aging, more than half of Chinese elderly at different age stages have obvious loneliness[3], which indicates that the overall health status of Chinese elderly is not optimistic. The low health level of the elderly would not only reduce the quality of life of individuals and families, but also bring heavy economic burden to the society[4].

Improving health is one of the important policy objectives of health insurance, especially social health insurance. A large number of studies have evaluated the health effects of health insurance carried out in different countries. However, existing studies have not reached a unanimous conclusion. Some studies believe that the expansion of health insurance coverage can significantly improve the health of participants and reduce the risk of death. Card et al. (2009) found that after joining Medicare, the mortality rate of the insured elderly would decrease significantly[5]. Chou et al. (2014) studied the impact of National Health Insurance (NHI) on infant mortality and found that the infant mortality rate in rural areas decreased by $8-16 \%$ after the implementation of NHI[6]. Kwack et al. (2013) also studied the impact of NHI on mortality, self-rated health and physical 
1 function limitations of the elderly, and found that NHI only reduced the risk of death of the elderly

2 group, and the most unhealthy group benefited the most[7]. Tian et al., (2012) found that people

3 without health insurance were more likely to suffer from severe depression risk[8]. Yu et al. (2019)

4 found that participating in any kind of health insurance would significantly improve the health of

5 the elderly, possibly due to the improvement of medical consumption level and the change of health behavior[9]. But other studies have shown that medical insurance has little or no significant effect on health. The famous RAND Medicare experiment found no significant difference in health

outcomes for individuals with different levels of reimbursement [10]. A study of low-income group population in India found no significant improvement in health outcomes despite increased healthcare utilization by insured people for the poor [11].

Since 1998, China has gradually established social medical insurance programs for different groups, including Urban Employee's Basic Medical Insurance (UEBMI) for urban employees, New Cooperative Medical Scheme (NCMS) for rural residents and Urban Resident Basic Medical Insurance (URBMI) for urban residents. Studies have evaluated the health effects of them respectively. Studies found that UEBMI could improve short-term and long-term health of participants to a certain extent[12], and it can significantly improve the health level of the elderly floating population[13]. The implementation of NCMS improved activities of daily living (ADL) and cognitive function, but did not significantly improve self-rated health[14]. Studies about the URBMI found URBMI enrollees had better health outcomes and the effect was stronger for those with lower education levels and lower income[15], and URBMI has a direct health improvement effect on the labor force[16]. However, other studies found that UEBMI has little effect on selfrated health of participants[17]. Some studies shown that NCMS had no significant effect on 
maternal and child mortality[18], and it did not significantly improve the self-rated health and illness or injury in the past four weeks for the enrollees[19, 20].

By comparing the health improvement effect of different types of basic medical insurance, studies found that the health effect of different medical insurance on the insured has obvious differences[13, 21]. UEBMI enrollees had better self-rated health, physical functioning and mental health than URBMI and NCMS participants[22]. UEBMI can promote the health of migrant workers, but URBMI and NCMS did not significantly improve their health effects[23]. In addition, Huang \& Wu (2020) also found that the integration of URBMI and NCMS had limited impact on the health of middle-aged and elderly rural residents[24].

In order to further reduce the economic burden of disease of patients with serious diseases, China has implemented Catastrophic Medical Insurance (CMI) since 2012. Residents who participate in URBMI and NCMS automatically participate in CMI without additional payment. After the reimbursement of basic medical insurance, CMI would provide extra compensation for those who still need to pay high medical expenses by themselves. Studies on CMI mainly focused on whether it could reduce economic risks and found CMI reduced the incidence of CHE[25-27]. Some studies have found that CMI has a limited role in reducing CHE[28], and it may even increase CHE intensity[29]. Currently, there is little literature exploring the health effects of CMI, Zhao et al. (2020) found that the implementation of CMI could significantly improve the health status of rural residents[30]. Huang \& Fu (2021) studied the impact of CMI implementation on mortality in the elderly[31]. Some studies discussed the impact of medical insurance integration model on the health of patients with serious diseases[32, 33].

Compared with previous studies, this study mainly aims to answer the following three 
1 questions :(1) what is the impact of CMI on the health status of the elderly? (2) Whether there is

2 heterogeneity in the health effects of CMI, whether there are differences in the effects of CMI for

3 the elderly with different residence and income levels. (3) What are the potential mechanisms behind

4 the effects of CMI on health?

\section{Methods}

\section{Data and study sample}

The data used in the study are from Chinese Longitudinal Healthy Longevity Survey (CLHLS),

which is combined conducted by Peking University and China Scientific Research Center on Aging.

This survey takes the elderly aged 65 and above as the object, aiming to explore the determinants of health and longevity of the elderly population in China. The design of the survey is scientific and representative[34, 35]. The CLHLS baseline survey was conducted in 1998, followed up in 2000, 2002, 2005, 2008/2009, 2011/2012, 2014, and 2018. The baseline and follow-up surveys covered 23 provinces, autonomous regions and municipalities in China. Considering the purpose of the study, the data of 2011/2012 and 2014 were selected as study samples, and data of other years were not selected, mainly for the following reasons.

China has reconstructed its medical insurance system covering different people since 1998, including UEBMI implemented in 1998, NCMS piloted in 2003, and URBMI piloted in 2007 and fully implemented in 2009. It can be seen that before 2009, Medical insurance system is to improve the coverage. In 2012, in order to further ease the medical economic burden of residents, the Chinese government implemented CMI with additional reimbursement for residents participating in NCMS and URBMI. The implementation time of CMI is not consistent in different regions, which enables 
us to use the framework of quasi-natural experiment to analyze the effect of CMI. To be specific, some provinces began to implement CMI in 2013, and most provinces began to implement CMI in 2014 and later. Therefore, the former can be used as the treatment group and the latter as the control group to analyze the effect of CMI. The reason why 2018 wave were not used is that in 2016 , China started the integration of URBMI and NCMS to promote the equitable enjoyment of medical security treatment for urban and rural residents. Therefore, the survey data of 2018 were not included in this study to avoid potential bias. The CMI is mainly for residents in URBMI and NCMS, and hence we screened out the original data of people who had participated in these two types of insurance. After sorting out and removing the samples with missing key variables, the final samples used in this study were 4727, including 551 and 4176 samples in the treatment group and control group, respectively.

\section{Measurements}

\section{Health outcome variables}

Multiple health indicators were used to measure health outcomes among the elderly, including self-rated health, Activities of Daily Living (ADL), Instrumental Activity of Daily Living (IADL), Mini-mental State Examination (MMSE), Index of Positive Well-Being and Index of Negative Well-Being.

Self-rated health, the most common health measure in household survey data, has been widely used in the existing studies[23, 36]. This study also adopted self-rated health as one of the health measures, which was based on a 5-level Likert Scale: How do you rate your health at present? The responses include $1=$ very good, $2=$ good, $3=$ so so, $4=$ bad, $5=$ very bad.

ADL were reflected in 6 aspects in CLHLS questionnaire, namely eating, taking a bath, dressing, 
1 going to the toilet, controlling urine and defecation, indoor transfer. The respondents would be asked

2 whether they needed help in each aspect, and there were three options for each aspect, including

3 being able to do it on your own without help, with partial help, with complete help. We construct 6

4 binary indicator for 6 aspects above, recoded as $0=$ can do it without help and $1=$ can't do it without

5 help, and the ADL variables used in this study was number of ADL limitations obtained by summing

6 up the six binary variables[37].

7 Similarly, IADL was measured by 8 questions in the CLHLS questionnaire, including visiting

8 the neighbor's house alone, going out shopping alone, cooking alone, washing clothes alone,

9 walking for 2 miles continuously, lifting $5 \mathrm{~kg}$ weight, squatting and standing up for 3 times

continuously, and taking transportation alone. There are three options for each aspect, including yes

11 and independently, yes but need some help, no and can't. By constructing 8 dummy variables, assign

the values of the last two options to 1 , and assign the values of the first option to 0 . Then we summed up the 8 binary variables to obtain number of IADL limitations used in this study.

Referring to existing studies[14], we used MMSE to measure individual cognitive function.

MMSE includes 13 questions of 4 aspects, i.e., orientation, registration, attention and calculation, recall and language. Each question has three options, namely correct, wrong and not able to answer.

We recoded the answer "not able to answer" as wrong based on existing research [38, 39]. Finally, a continuous variable MMSE with a value range of $0-30$ points was obtained by summing up all the related variables. Individuals with higher MMSE scores had better cognitive function. 
1 at present, with $1=$ very bad, $2=$ bad, $3=$ so so, 4=good, 5=very good; Do you always look on the

2 bright side of things, with $1=$ always, $2=$ often, $3=$ sometimes, $4=$ seldom, $5=$ never; Are you as happy

3 as when you were younger, with $1=$ always, $2=$ often, $3=$ sometimes, $4=$ seldom, 5=never. The

4 answers to these three questions were added together to obtain the index of positive well-being

5 ranging from 3 to 15 , which is the positive health variable used in our study. Individuals with higher index had better mental health. Negative mental health involves three related questions, including

7 Do you often feel fearful or anxious? Do you often feel lonely and isolated? Do you feel the older 8 you get, the more useless you are, and have trouble doing anything? Each of the above three

9 questions has five options: 1 = always, $2=$ often, $3=$ sometimes, $4=$ seldom, $5=$ never. The negative score ranging from 3-15 were obtained by summing up the three variables. Individuals with lower index of negative well-being had better mental health.

\section{CMI}

The key explanatory variable in this study was CMI, which is whether an area has implemented

CMI in the past year. To construct this variable, we collected the policy documents issued by provinces and cities to implement CMI, and matched them with CLHLS data sets at the individual level to explore the impact of CMI on health among elderly. The policy documents of different regions from the official websites of each regional government or the medical insurance administration. Our judgment is based on the year in which each province started to fully implement CMI. The time distribution of each province implementing CMI was shown in figure1. Finally, we selected five provinces as the treatment group, namely Liaoning, Jilin, Fujian, Hubei and Chongqing, and took the other provinces as the control group. 


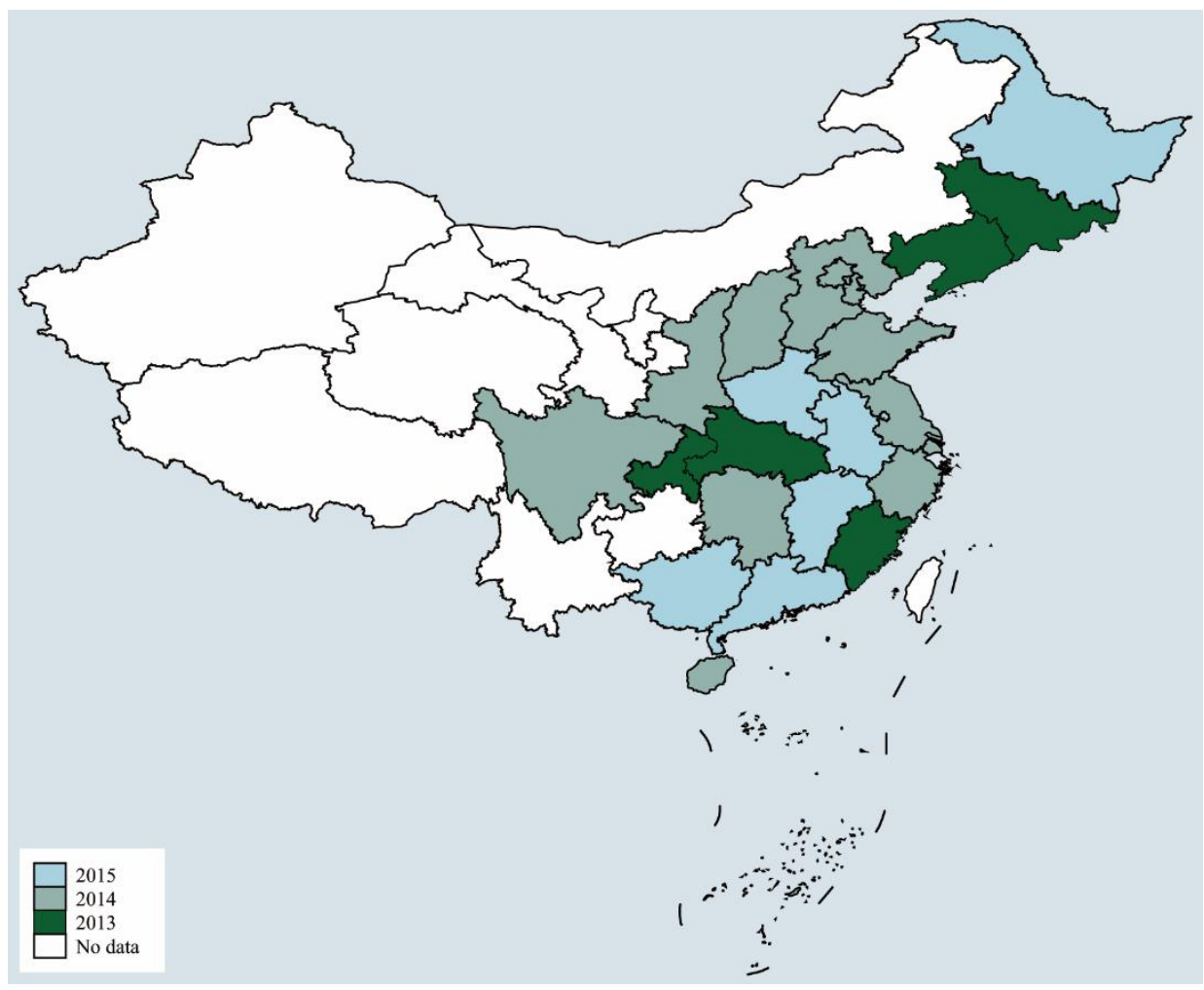

Figure1. The implementation time of CMI among the 23 provinces (municipalities,

autonomous regions) in CLHLS.

\section{Covariates}

In this study, we controlled for variables that might confuse the relationship between health outcomes and CMI, with variable selection based primarily on existing studies[14, 22] and data availability. Covariates included the following categories: sociodemographic characteristics, socioeconomic status, and health-related behaviors. Sociodemographic characteristics included age, gender, marital status and number of children, and whether the elderly live alone. Marital status is divided into two categories, with 0 indicating the respondent was divorced, widowed or never married, 1 indicating the elderly was married. Whether the elderly live alone or not is a dummy variable, 0 represents the respondent live with his/her family. 1 indicated the elderly lived alone.

Socioeconomic status variables included years of education, type of job held before age 60 , household income per capita(logarithmic), and place of residence, where the job type before the age 
of 60 is a dummy variable ( $1=$ had a white-collar job, $0=$ others $)$. There are two categories of residence, where 0 means an individual lives in a rural area and 1 means an individual lives in an urban area. Regarding the health-related behaviors, we controlled 3 variables: Smoking (1=smoke at the present $)$ and Drinking ( $1=$ drink alcohol at the present). Considering that chronic diseases is degenerative diseases, the number of chronic diseases suffered by the elderly is also included in our study as a control variable.

\section{Statistical Methods}

\section{Difference-in-Differences model}

As mentioned before, the implementation time of CMI in different provinces (municipalities directly under the Central Government and autonomous regions) is inconsistent, so we adopted difference-in-differences model to study the impact of CMI on health outcomes of the elderly, and take it as the benchmark. The specific model is set as follows.

$$
y_{i p t}=\alpha+\beta_{0} \cdot \text { time }_{t}+\beta_{1} \cdot \text { treat }_{p}+\delta \cdot\left(\text { treat }_{p} \times \text { time }_{t}\right)+\boldsymbol{Z}_{\text {ipt }}^{\prime} \boldsymbol{\eta}+\sigma_{p}+\varepsilon_{\text {ipt }} .(1)
$$

Where $y_{\text {ipt }}$ represented the health status of individual $i$ of province $p$ in period $t$. time $e_{t}$ is a binary variable, with 0 indicated that the period belongs to 2011/2012, 1 indicated that the observations belongs to 2014. treat $_{p}$ is also a dichotomized variable, with $1=$ the individual belongs to the provinces where CMI is implemented, including Liaoning, Jilin, Fujian, Hubei and Chongqing, 0 indicated that the individual belongs to the provinces where CMI is implemented in 2014 or later. The coefficient of the interaction term of treat $t_{p}$ and time $_{t}$ is the effect we are concerned with, namely, the effect of CMI on the health of the elderly. $\boldsymbol{Z}_{\boldsymbol{i p t}}^{\prime}$ represented other covariates that may affect individual health; $\sigma_{p}$ is the provincial fixed effect used to control for confounding factors at the provincial level; $\varepsilon_{i p t}$ is the error term of the model. 
In addition, in order to answer our second question, we added interaction items of treat $t_{p}$, time $_{t}$ and residence, income variable in the model (1) to verify the CMI effect differences to the health of urban and rural residents and different income group population (model (2)).

$$
\begin{aligned}
& y_{i p t}=\alpha+\beta_{0} \cdot \text { time }_{t}+\beta_{1} \cdot \text { treat }_{p}+\delta \cdot D I D_{i p t}+\delta_{1}\left(D I D_{i p t} \cdot \operatorname{Rural}_{i p t}\right)+\delta_{1}\left(D I D_{i p t}\right.
\end{aligned}
$$

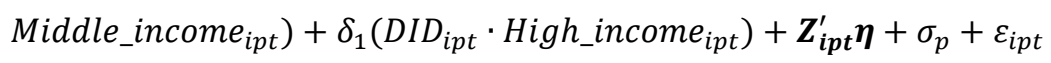

Where $D I D_{i p t}$ is the treat $_{p} \times$ time $_{t}$ in model (1). Urban $i p t$ is a dummy variable, where 1 means that individuals live in cities and towns, and 0 means that individuals live in rural areas. Therefore, coefficient $\delta_{1}$ reflected the difference of health impact of CMI on urban and rural residents. In order to further study the influence of CMI on the health of individuals in different income group population, we divided the sample into three categories according to the income quantile of the sample, and generated three corresponding binary variables. Considering that there would be complete collinearity if all of them were added into the model, we took the low-income group as the reference group and added the interaction terms of $D I D_{i p t}$ and Middle_income $e_{i p t}$, $D I D_{i p t}$ and High_income ${ }_{i p t}$, respectively to observe the impact of CMI on the health of the elderly with different income levels.

\section{PSM-DID}

To make individuals more comparable between treatment and control groups, we used propensity score matching-difference-in-differences (PSM-DID) method based on the benchmark[14] and take it as the robustness test. To be specific, we first screened the sample and set it as balanced panel data, that is, every individual in the sample was interviewed in both two waves. Then, the sample of the former wave (2011/12) and the covariate mentioned above were 
used to establish logit model to obtain propensity score $\left(P\left(\right.\right.$ treat $\left.\left.=1 \mid Z_{i}\right)\right)$, and the matching of individuals between the treatment and control group in the common support was carried out by the kernel matching strategy. Then the matched data and DID model were employed to estimate the impact of CMI on the health of the elderly. Finally, the average treatment effects on the treated was as follows.

(1)

$$
\begin{gathered}
A T T=\mathrm{E}\left(Y_{i, 2014}^{\text {treat }}-Y_{i, 2011 / 12}^{\text {treat }} \mid P\left(\text { treat }=1 \mid Z_{i}\right), \text { treat }=1\right)-\mathrm{E}\left(Y_{i, 2014}^{\text {control }}-\right. \\
\left.Y_{i, 2011 / 12}^{\text {control }} \mid P\left(\text { treat }=1 \mid Z_{i}\right), \text { treat }=0\right)
\end{gathered}
$$

\section{Heckman sample selection model}

To estimate the potential mechanisms of the impact of CMI on individual health, we used the Heckman selection model to analyze the impact of CMI on healthcare costs over the past year[41].

The model consists of two stages. The first stage is the selection stage. The selection equation, namely a probit model, was used to predict the probability of individual seeking medical treatment, and the inverse Mills ratio of each observation was calculated, which reflected the instantaneous probability of selecting each observation into the sample. Two variables were included in our study, whether the respondents had seen a doctor in the past year, and whether the respondents were hospitalized in the past year. the second stage is the outcome stage, and a linear regression was employed at this stage to predict the total medical expenses (including the out-of-pocket and reimbursement payment) and the hospitalization expenses (including the out-of-pocket and reimbursement payment) in the past year. The dependent variables were the natural logarithm of them, and the inverse mills ratio was added to the second-stage regression to control for selection bias (there are systematic differences between those individuals who choose to see a doctor or be hospitalized and those who do not). When there is a significant correlation between the error terms 
of the selection equation and the result equation, the estimation results of only linear regression results would have a bias, indicating that it must be corrected[41].

\section{Results}

\section{Characteristics of the study population}

Table 1 compared the characteristics of individuals in CMI implementation and nonimplementation groups. There were 551 and 4176 elderly people in the treatment and control group, respectively. From the perspective of health outcomes, most of the elderly in the whole sample reported general health status (39\%), followed by those who believed good (35\%). In terms of activities of daily living (ADL) and Instrumental Activity of Daily Living (IADL), both types of limitations were worse in the control group. In addition, there was a significant difference in IADL between the two groups $(\mathrm{P}<0.01)$. MMSE score, which reflected individual cognitive function, showed that the cognitive function of the elderly in the treatment group was slightly worse than that in the control group (25.66 vs. 26.09), and the difference was significant at the level of $10 \%(\mathrm{P}<0.1)$.

From the results of the indices of positive and negative well-being, there was significant difference in index of positive well-being between the two groups $(\mathrm{P}<0.05)$, but there was no significant difference in index of negative well-being between them $(\mathrm{P}>0.1)$. The average age of the elderly in the whole sample was $82.13 \pm 9.61$ years old, and years of education of the elderly in the treatment group was significantly higher than that in the control group (2.41 vs. 2.08). Also, individuals in the treatment group had more chronic diseases than those in control group (1.83 vs. 1.58). In addition, there were significant differences between the treatment group and the control group in medical insurance type, residence and smoking $(\mathrm{P}<0.05)$.

Table1 Characteristics of respondents

Control group Treatment group Total




\begin{tabular}{|c|c|c|c|}
\hline Variables & $(\mathrm{N}=4176)(\% /$ mean $(\mathrm{sd}))$ & $(N=551)$ & $(\mathrm{N}=4727)$ \\
\hline \multicolumn{4}{|l|}{ Self-rated Health $(\%)^{\text {a }}$} \\
\hline Very good & $11 \%$ & $10 \%{ }^{*}$ & $11 \%$ \\
\hline Good & $35 \%$ & $33 \%$ & $35 \%$ \\
\hline So so & $38 \%$ & $44 \%$ & $39 \%$ \\
\hline Bad & $14 \%$ & $11 \%$ & $14 \%$ \\
\hline Very bad & $1 \%$ & $1 \%$ & $1 \%$ \\
\hline Number of ADL limitations & $0.25(0.82)$ & $0.21(0.74)$ & $0.25(0.81)$ \\
\hline Number of IADL limitations & $2.11(2.62)$ & $1.77(2.53)^{\star \star \star}$ & $2.07(2.61)$ \\
\hline MMSE score & $26.09(4.90)$ & $25.66(5.52)^{\star}$ & $26.04(4.98)$ \\
\hline Index of Positive Well-Being & $11.20(2.09)$ & $11.13(1.98)^{* *}$ & $11.20(2.08)$ \\
\hline Index of Negative Well-Being & $6.68(2.23)$ & $6.60(2.26)$ & $6.67(2.24)$ \\
\hline Age (years old) & $82.23(9.67)$ & $81.32(9.14)$ & $82.13(9.61)$ \\
\hline Years of Schooling & $2.03(2.95)$ & $2.41(3.12)^{\star \star *}$ & $2.08(2.97)$ \\
\hline Number of Children & $3.94(1.77)$ & $4.05(1.76)$ & $3.95(1.77)$ \\
\hline Household Income Per Capita (logarithm) & $8.43(1.42)$ & $8.45(1.55)$ & $8.43(1.44)$ \\
\hline Number of Chronic Disease & $1.58(1.49)$ & $1.83(1.58)^{* * *}$ & $1.61(1.51)$ \\
\hline Female (\%) & $55 \%$ & $54 \%$ & $54 \%$ \\
\hline Had a White-collar Job before $60(\%)$ & $3 \%$ & $3 \%$ & $3 \%$ \\
\hline Rural China (\%) & $54 \%$ & $46 \%{ }^{* \star *}$ & $53 \%$ \\
\hline Married (\%) & $46 \%$ & $49 \%$ & $46 \%$ \\
\hline Live Alone & $19 \%$ & $17 \%$ & $19 \%$ \\
\hline NCMS (\%) & $90 \%$ & $82 \%{ }^{\star \star \star}$ & $89 \%$ \\
\hline Smoking (\%) & $21 \%$ & $25 \%$ ** & $21 \%$ \\
\hline Drinking (\%) & $19 \%$ & $19 \%$ & $19 \%$ \\
\hline
\end{tabular}

1 a For continuous variables, $\mathrm{T}$ test was used to see whether the differences of variables were significant between the treatment and control group; for categorical variables, Chi-square test was used to see whether the differences of variables were significant between the two groups.

$3 \quad{ }^{*} P<0.1{ }^{* *} P<0.05{ }^{* * *} P<0.01$

Table 2 showed the main DID regression results of the impact of CMI on health among the

6 Chinese elderly. It can be seen from the model that, after controlling information at the provincial

7 level and other covariates of individuals, the implementation of CMI can improve the self-rated

8 health of the elderly by 0.065 units, reduce the limited number of IADL by 0.132 , increase the

9 limited number of ADL by 0.012 , and decrease the MMSE score by 0.494 points. However, these

10 effects are not statistically significant $(P>0.1)$. It can be seen that, CMI could increase the index of

11 positive well-being by 0.391 units and decrease the index of negative well-being by 0.370 units, 
1 which were both significant at the level of $5 \%(P<0.05)$.

2

Table2 Main results: the impact of CMI on health of the elderly in China

\begin{tabular}{|c|c|c|c|c|c|c|}
\hline Variables & $\begin{array}{c}\text { Self-rated } \\
\text { Health }\end{array}$ & $\begin{array}{c}\text { Number of ADL } \\
\text { limitations }\end{array}$ & $\begin{array}{c}\text { Number of IADL } \\
\text { limitations }\end{array}$ & $\begin{array}{l}\text { MMSE } \\
\text { score }\end{array}$ & $\begin{array}{l}\text { Index of Positive } \\
\text { Well-Being }\end{array}$ & $\begin{array}{c}\text { Index of Negative } \\
\text { Well-Being }\end{array}$ \\
\hline \multirow[t]{2}{*}{ treat $_{p}$} & $0.438^{\star * *}$ & -0.251 & $-0.723^{\star}$ & -0.826 & $-1.489^{\star \star \star}$ & $0.926^{* \star *}$ \\
\hline & $(0.137)$ & $(0.156)$ & $(0.396)$ & $(0.791)$ & $(0.316)$ & $(0.330)$ \\
\hline \multirow[t]{2}{*}{ time $_{t}$} & -0.019 & 0.012 & 0.046 & $0.365^{\star \star \star}$ & -0.099 & $0.131^{\star *}$ \\
\hline & $(0.026)$ & $(0.023)$ & $(0.062)$ & $(0.131)$ & $(0.064)$ & $(0.065)$ \\
\hline \multirow[t]{2}{*}{$D I D_{i p t}$} & -0.065 & 0.012 & -0.132 & -0.494 & $0.391^{\star *}$ & $-0.370^{* *}$ \\
\hline & $(0.072)$ & $(0.062)$ & $(0.165)$ & $(0.370)$ & $(0.173)$ & $(0.177)$ \\
\hline Province & Yes & Yes & Yes & Yes & Yes & Yes \\
\hline Covariates & Yes & Yes & Yes & Yes & Yes & Yes \\
\hline Observations & 4,727 & 4,727 & 4,727 & 4,727 & 4,727 & 4,727 \\
\hline Adjusted $R^{2}$ & 0.130 & 0.102 & 0.367 & 0.219 & 0.075 & 0.080 \\
\hline
\end{tabular}

$3 \quad{ }^{*} P<0.1{ }^{* *} P<0.05^{* * *} P<0.01$

4 In order to further examine the impact of CMI on different subgroups, we added the interaction

5 item of $D I D_{\text {ipt }}$ and residence Rural $_{i p t}$, and interaction items of $D I D_{i p t}$ and the binary variables

6 Middle_income $e_{i p t}, H_{i g h}$ income $_{\text {ipt }}$. It can be seen that the impact of CMI on self-rated health,

7 IADL and MMSE Score were still insignificant $(P>0.1)$. But CMI could significantly reduce the

8 number of ADL limitations of the elderly living in rural areas and middle- and high-income

$9 \operatorname{groups}(P<0.1)$. In addition, $\mathrm{CMI}$ could significantly increase the index of positive well-being and

10 reduce the index of negative well-being of high-income group population $(P<0.1)$

11

Table 3 Heterogeneity analysis

\begin{tabular}{|c|c|c|c|c|c|c|}
\hline Variables & $\begin{array}{c}\text { Self-rated } \\
\text { Health }\end{array}$ & $\begin{array}{c}\text { Number of } \\
\text { ADL limitations }\end{array}$ & $\begin{array}{l}\text { Number of } \\
\text { IADL } \\
\text { limitations }\end{array}$ & MMSE score & $\begin{array}{c}\text { Index of } \\
\text { Positive Well- } \\
\text { Being }\end{array}$ & $\begin{array}{c}\text { Index of } \\
\text { Negative Well- } \\
\text { Being }\end{array}$ \\
\hline \multirow[t]{2}{*}{ treat $_{p}$} & $0.431^{* * *}$ & $-0.263^{*}$ & $-0.751^{*}$ & -0.833 & $-1.486^{\star * *}$ & $0.928^{\star * *}$ \\
\hline & $(0.137)$ & $(0.157)$ & $(0.396)$ & $(0.792)$ & $(0.316)$ & $(0.330)$ \\
\hline \multirow[t]{2}{*}{ time $_{t}$} & -0.019 & 0.011 & 0.045 & $0.364^{\star \star *}$ & -0.096 & $0.128^{\star *}$ \\
\hline & $(0.026)$ & $(0.023)$ & $(0.062)$ & $(0.131)$ & $(0.064)$ & $(0.065)$ \\
\hline \multirow[t]{2}{*}{$D I D_{i p t}$} & 0.036 & $0.342^{*}$ & 0.189 & -0.247 & -0.032 & 0.017 \\
\hline & $(0.126)$ & $(0.176)$ & $(0.332)$ & $(0.730)$ & $(0.298)$ & $(0.296)$ \\
\hline \multirow[t]{2}{*}{$D_{I D} \cdot$ Rural $_{i p t}$} & -0.111 & $-0.227^{\star \star}$ & -0.394 & -0.144 & 0.207 & -0.129 \\
\hline & $(0.105)$ & $(0.111)$ & $(0.267)$ & $(0.610)$ & $(0.251)$ & $(0.282)$ \\
\hline \multirow[t]{2}{*}{$D_{I} D_{\text {ipt }} \cdot$ Middle_income $e_{\text {ipt }}$} & 0.017 & $-0.348^{\star \star}$ & -0.271 & -0.346 & 0.044 & -0.179 \\
\hline & $(0.125)$ & $(0.146)$ & (0.328) & $(0.853)$ & $(0.309)$ & $(0.330)$ \\
\hline
\end{tabular}




\begin{tabular}{|c|c|c|c|c|c|c|}
\hline & $(0.123)$ & $(0.156)$ & $(0.325)$ & $(0.702)$ & $(0.295)$ & $(0.320)$ \\
\hline Covariates & Yes & Yes & Yes & Yes & Yes & Yes \\
\hline
\end{tabular}

\section{Robustness test}

Although the DID model controlled as many factors as possible, due to the limitations of two

4 periods of data, we could not verify whether the assumptions of DID method, namely common trend

5 was met. Therefore, we referred to the practices of existing studies.[14] The results of PSM-DID

6 model were used as the robustness test of our benchmark results. Firstly, the samples were screened

7 into panel data, and then the samples were matched according to the characteristics of the baseline

8 survey (2011/12 wave). Then the effect of CMI on health of the elderly was studied using the DID

9 method. Table A1 and Figure A1 list the balance test results and the kernel density curve of

10 propensity score before and after matching. It can be seen from Panel A in Table A1 that after

11 matching, all observable characteristics between the treatment and control group were well balanced.

12 Variables with significant differences between the two groups became statistically insignificant

$13(P>0.05)$ after matching.

14

Table 4 Robust test

\begin{tabular}{|c|c|c|c|c|c|c|}
\hline Variables & Self-rated Health & $\begin{array}{c}\text { Number of } \\
\text { ADL limitations }\end{array}$ & $\begin{array}{l}\text { Number of } \\
\text { IADL } \\
\text { limitations }\end{array}$ & $\begin{array}{l}\text { MMSE } \\
\text { score }\end{array}$ & $\begin{array}{c}\text { Index of } \\
\text { Positive Well- } \\
\text { Being }\end{array}$ & $\begin{array}{c}\text { Index of } \\
\text { Negative Well- } \\
\text { Being }\end{array}$ \\
\hline$D I D_{i p t}$ & $\begin{array}{l}-0.117^{* *} \\
(0.059)\end{array}$ & $\begin{array}{l}-0.050 \\
(0.077)\end{array}$ & $\begin{array}{l}-0.080 \\
(0.175)\end{array}$ & $\begin{array}{c}0.253 \\
(0.394)\end{array}$ & $\begin{array}{c}0.094 \\
(0.142)\end{array}$ & $\begin{array}{l}-0.375^{\star *} \\
(0.151)\end{array}$ \\
\hline Province & Yes & Yes & Yes & Yes & Yes & Yes \\
\hline Covariates & Yes & Yes & Yes & Yes & Yes & Yes \\
\hline Observations & 3,928 & 4,078 & 4,210 & 3,250 & 3,460 & 3,646 \\
\hline Adjusted $R^{2}$ & 0.045 & 0.084 & 0.146 & 0.013 & 0.008 & 0.011 \\
\hline
\end{tabular}

${ }^{*} P<0.1{ }^{* *} P<0.05$ *** $P<0.01$

16 Meanwhile, Panel B in table A1 showed the overall balancing properties of kernel matching. 
the Pseudo R square of probability model, the joint significance of covariates, the mean and median of the standardized deviation were all significantly lower after matching. As can be seen from the probability distribution density function in Figure A1, the probability distribution density curves of the two groups were close to coincidence after matching, indicating that the samples of the treatment and control group were more balanced after matching.

Table 4 reported the PSM-DID estimation results. It can be seen that CMI could significantly improve self-rated health and index of negative well-being among the elderly. Specifically, the selfrated health of the elderly in treatment group was improved by 0.117 units, and the index of negative well-being decreased by 0.375 units in the treatment group, and the above effects were significant at the level of 5\% $(P<0.05)$. Moreover, the estimation results showed that CMI could reduce the number of ADL and IADL limitations, and increase MMSE score and index of positive well-being by 0.253 and 0.094 points, respectively. However, these effects were not statistically significant $(P>0.1)$.

\section{Potential Mechanisms analysis}

In order to verify the potential mechanisms of CMI influencing the health of the elderly, we used the Heckman Selection model to estimate the impact of CMI on the total healthcare utilization and inpatient healthcare utilization of the elderly. The results are shown in Table 5. for the total healthcare utilization, the inverse Mills ratio was significant at the level of $1 \%$, indicating that there was an obvious selection effect in patients' decision making. But there was no significant selection effect on hospitalization. After controlling for provincial information and related covariates, we found that CMI mainly increased the hospitalization rate of the elderly $(P<0.01)$, but did not significantly affect their total hospitalization expenditure, probability of visit, and total medical 
expenditure $(P>0.1)$.

\section{Discussion}

In order to reduce the risk of catastrophic health expenditure for urban and rural residents, China has issued a document to establish and promote the implementation of the catastrophic medical insurance since 2012. The gradual implementation of CMI in different regions provides a good basis for studying the effect of the implementation of it. Existing studies discussed the effect of CMI in lowering medical economic risks, ignoring its influence on health outcomes. The study based on a natural experiment framework, using the CLHLS data and a number of health indicators, evaluated the effect of CMI on health outcomes among the elderly and its heterogeneity. Also, its potential mechanism was studied. The conclusion can provide useful references for improving the medical insurance system design in China and other developing countries.

Our study found that the implementation of CMI can improve the mental health status of the elderly to some extent, which is consistent with the conclusions of previous studies[31]. From the perspective of system design, CMI mainly compensates for the compliance medical expenses that still need to be paid by individuals after being reimbursed by the basic medical insurance. Studies have pointed out that CMI can reimburse an additional $10 \%$ of the expenses on the basis of the basic medical insurance[42]. This medical cost sharing mechanism of CMI can further improve the accessibility of hospitalization services and hence increase the probability of hospitalization. One study found that CMI increased the frequency and length of hospital stays, promoted the utilization of medical services for inpatients[26]. In addition, CMI could promote the subjective healthcare accessibility of the elderly, increases the probability of timely treatment when the elderly was ill[31], the promotion in healthcare utilization accessibility could improve the health outcomes of the 
elderly, especially in the aspect of mental health.

Our study also demonstrated that CMI had a limited effect on the health improvement of the elderly. CMI had no significant impact on the physical health of the elderly, including ADL, IADL and cognitive function. Existing studies on the effect of URBMI and NCMS found individuals would be excluded from the scope of compensation due to high deductibles, Low reimbursement rate and limited coverage[20]. Therefore basic medical insurance contribute only modestly to health improvements[23]. CMI provides extra compensation for URBMI and NCMS patients with high medical costs. Although the level of security is improved to a certain extent, the protection effect is limited[43], which may also be the reason for the limited health improvement effect. Moreover, since health is a stock, the impacts of the cost-sharing mechanism of CMI on health outcomes of the elderly deserve further study[17].

Finally, our study showed that there was heterogeneity in the health improvement effect of CMI among different groups. The positive effect of CMI on health was mainly concentrated in the elderly with higher income level, and there is no significant improvement effect on the middle- and low-income group, especially for the low-income group population. The coverage of CMI and its segmented compensation design suggested that people who pay more out-of-pocket medical expenses get more subsidies from it. As studies have shown, out-of-pocket health expenditures increase as affordability increases[44]. For individuals, in order to get additional compensation from CMI, they have to reach the high deductible. For the low-income group population, to achieve the threshold means that they need to undertake a certain proportion of out-of-pocket medical expenses. And those constrained by their income could not enjoy CMI benefits because it was hard to pay the threshold for them. A study about the NCMS also found that participants with low- and middle- 
income were more likely to avoid using medical services[20]. Therefore, higher income group will benefit more from CMI[26]. In contrast, the benefits of the CMI are not fully available to lowincome group population, so there is no significant improvement in health outcomes. The income heterogeneity of the health effect of critical illness insurance is consistent with previous studies on the general population[30].

Our study had several policy implications. First of all, CMI should focus on its health improvement effect in the future since the ultimate goal of health insurance is to improve the health outcomes of participants. For patients with serious diseases, the government should focus on their health demand and give personalized medical insurance reimbursement plan; Second, CMI should take fairness into consideration during the process of practice, focusing on welfare improvement for low-income group population. Although CMI has provided some priorities in view of the extreme poverty population in the process of implementation, such as to reduce the deductible, cancel the cap line, increase reimbursement ratio by $5-10 \%$ and so on. However, some people with lower income level are still excluded from the scope of policy security due to budget constraints. In view of this problem, it is suggested to design the compensation policy of CMI according to the gradient of residents' disposable income, so as to make the policy security more accurate. Finally, although most areas are introduced CMI mainly for hospitalized patients with serious illness, but for some patients with specific diseases, the outpatient medical expenses are also burdensome. These people remain at high risk of falling into catastrophic health spending. Therefore, it can be considered to broaden CMI coverage, but need to pay attention to individual excessive demand and induced demand from staff in health facilities.

Our study had some limitations. Firstly, considering the implementation of CMI to the 
1 improvement of individual health needs some time, but data used in this study cannot capture the

2 health effect of CMI for a long time, so this is one of the limitations of this study, and it is also a

3 research direction in the future. Secondly, the influence of CMI on health of other group population

4 is also a research direction we will pay attention to in the future.

\section{Conclusions}

6 This study analyzed the impact of CMI on the health among Chinese elderly. The results showed

7 that CMI could improve the mental health of the elderly to a certain extent, but the effect is limited.

8 CMI mainly improves the health of middle- and high-income groups, especially high-income group

9 population, and did not significantly affect any health outcomes of low-income elderly. The possible mechanism for the impact of CMI on health is that it can promote access to healthcare utilization

11 for older people through price subsidy mechanisms, especially increase the probability of hospitalization. Our research conclusions have important policy implications. The government should pay more attention to health improvement effect and its fairness in the future implementation process of CMI. In the premise to ensure the sustainable and effective regulation of the CMI fund, the reimbursement coverage can be expanded and the reimbursement rate of CMI can be increased appropriately, making CMI more accessible to those need it most.

\section{List of abbreviations}

18 CMI: Catastrophic medical insurance

CLHLS: Chinese Longitudinal Healthy Longevity Survey

DID: Difference-in-differences

PSM: Propensity score matching 
2 NCMS: New Cooperative Medical Scheme

3 NHI: National Health Insurance

4 ADL: Activities of Daily Living

5 IADL: Instrumental Activity of Daily Living

6 MMSE: Mini-mental State Examination

7 Acknowledgments

8 We thank the Center for Healthy Ageing and Development Studies, Peking University, very much

9 for providing the data of CLHLS.

10 Availability of data and materials

11 https://sites.duke.edu/centerforaging/programs/chinese-longitudinal-healthy-longevity-survey-

12 clhls/

13 Authors' Contributions

$14 \mathrm{XX}$ and HY designed the study. JS and XX led the data analysis and wrote the manuscript. HY, JS, XX

15 and QW participated in the revision of the manuscript and approved the final version for publication.

16 Ethics approval and consent to participate

17 Ethical approval for the study was not required since it was based exclusively on the publicly available

18 data, CLHLS. Hence the study subjects were not directly approached.

\section{Consent for Publication}

$20 \quad$ No applicable.

$21 \quad$ Competing interest

22 The authors report no conflicts of interest in this work. 
3 1. National Bureau of Statistics. Main data of the seventh national population census. 2021. http://www.stats.gov.cn/tjsj/zxfb/202105/t20210510_1817183.html. Accessed 18 Nov 2021.

2. National Health Commission of the People's Republic of China. 2019. Healthy China Action (2019-2030). http://www.nhc.gov.cn/guihuaxxs/s3585u/201907/e9275fb95d5b4295b e8308415d4cd1b2.shtml. Accessed 18 Nov 2021.

3. Dang J, Li J, Zhang Q, Luo X: Development of Report on the Quality of Life for the Elderly in China (2019). China Beijing: Social Sciences Academic Press (China); 2019. (In Chinese)

4. Feng J, Yu Y, Lou P: Medical Demand and Growing Medical Costs in China--Based on the

6. Chou S-Y, Grossman M, Liu J-T: The impact of national health insurance on birth outcomes: a natural experiment in Taiwan. Journal of Development Economics 2014, 111:75-91.

7. Keng SH, Sheu SJ: The effect of national health insurance on mortality and the SES-health gradient: evidence from the elderly in Taiwan. Health economics 2013, 22:52-72. on depression: an epidemiological cohort study of a randomized community sample in Northwest China. BMC psychiatry 2012, 12:1-11. 
and Health of the Elderly: An Evaluation of Institutional Effects and Analysis of Mechanisms. Financial Economics Research 2019, 34:149-160. (In Chinese)

10. Newhouse JP, Group RCIE, Corporation R, Staff IEG: Free for all?: lessons from the RAND health insurance experiment. Harvard University Press; 1993.

11. Shrestha R: Health Insurance for the Poor, Health Care Utilisation and Health Outcomes in Indonesia. Bulletin of Indonesian Economic Studies 2020:1-44.

12. Chen H, Deng P: Health Effect Evaluation of the Urban Employee Basic Medical Insurance. Social Security Studies 2016:44-52. (In Chinese) Meng Y, Han J, Qin S: The impact of health insurance policy on the health of the senior

14. Cheng L, Liu H, Zhang Y, Shen K, Zeng Y: The impact of health insurance on health outcomes and spending of the elderly: evidence from China's new cooperative medical scheme. Health economics 2015, 24:672-691. 
1 19. Lei X, Lin W: The New Cooperative Medical Scheme in rural China: does more coverage mean more service and better health? Health economics 2009, 18:S25-S46.

20. Ma X, Oshio T: The impact of social insurance on health among middle-aged and older adults in rural China: a longitudinal study using a three-wave nationwide survey. $B M C$ public health 2020, 20:1-9.

21. Su M, Zhou Z, Si Y, Wei X, Xu Y, Fan X, Chen G: Comparing the effects of China's three basic health insurance schemes on the equity of health-related quality of life: using the method of coarsened exact matching. Health and quality of life outcomes 2018, 16:1-12.

22. Liu X, Wong H, Liu K: Outcome-based health equity across different social health insurance schemes for the elderly in China. BMC health services research 2015, 16:1-12.

23. Qin X, Pan J, Liu GG: Does participating in health insurance benefit the migrant workers in China? An empirical investigation. China Economic Review 2014, 30:263-278.

24. Huang $\mathrm{X}, \mathrm{Wu} \mathrm{B}$ : Impact of urban-rural health insurance integration on health care: Evidence from rural China. China Economic Review 2020, 64:101543.

25. Zhang Y, Vanneste J, Xu J, Liu X: Critical Illness Insurance to alleviate catastrophic health expenditures: new evidence from China. International journal of health economics and management 2019, 19:193-212.

26. Jiang J, Chen S, Xin Y, Wang X, Zeng L, Zhong Z, Xiang L: Does the critical illness insurance reduce patients' financial burden and benefit the poor more: A comprehensive evaluation in rural area of China. Journal of medical economics 2019, 22:455-463.

27. Zhao S-w, Zhang X-y, Dai W, Ding Y-x, Chen J-y, Fang P-q: Effect of the catastrophic medical insurance on household catastrophic health expenditure: evidence from China. 
28. Fang P, Pan Z, Zhang X, Bai X, Gong Y, Yin X: The effect of critical illness insurance in China. Medicine 2018, 97.

29. Li A, Shi Y, Yang X, Wang Z: Effect of critical illness insurance on household catastrophic health expenditure: the latest evidence from the National Health Service Survey in China. International journal of environmental research and public health 2019, 16:5086.

30. Zhao W: Does New Rural Cooperative Medical Insurance Improve the Health of Rural Residents? . Journal of Finance and Economics 2020, 46:141-154.

31. Huang J, Fu H: The Impact of Supplemental Health Insurance on the Mortality Rate of the Elderly: Evidence from Critical Illness Insurance. The Journal of World Economy 2021, 44:179-200. (In Chinese)

32. Wang Q: Study on the Effect of Critical Illness Insurance System for Urban and Rural Residents. Journal of Public Management 2019, 16:96-107+173. (In Chinese)

33. Xu X: Urban-Rural Healthcare Pooling's Impacts on Healthcare Utilization and Health Inequality for Patients with Serious Illness-An Empirical study based on micro survey data of eastern, central and western China. Insurance Studies 2021:111-127. (In Chinese)

34. Wang J, Taylor A, Zhang T, Appleton S, Shi Z: Association between body mass index and all-cause mortality among oldest old Chinese. The journal of nutrition, health \& aging 2018, 22:262-268.

35. CfHAaD S: The Chinese Longitudinal Healthy Longevity Survey (CLHLS)-Longitudinal Data (1998-2018). Peking University Open Research Data Platform 2020.

36. Guan M: Associations between schemes of social insurance and self-rated health 

health 2019, 7:253.

37. Guo A, Gu D: The Association between Access to Healthcare and Health Outcomes among Older Adults in China from Health Disparity--Based on CLHLS Data. Population \& Development 2020, 26:60-69. (In Chinese)

38. Zhang Z: Gender differentials in cognitive impairment and decline of the oldest old in

China. The Journals of Gerontology Series B: Psychological Sciences and Social Sciences 2006, 61:S107-S115 China. Journal of family issues 2008, 29:1379-1403. 


\section{Appendix}

2

Table A1 The balancing tests of covariates from kernel matching

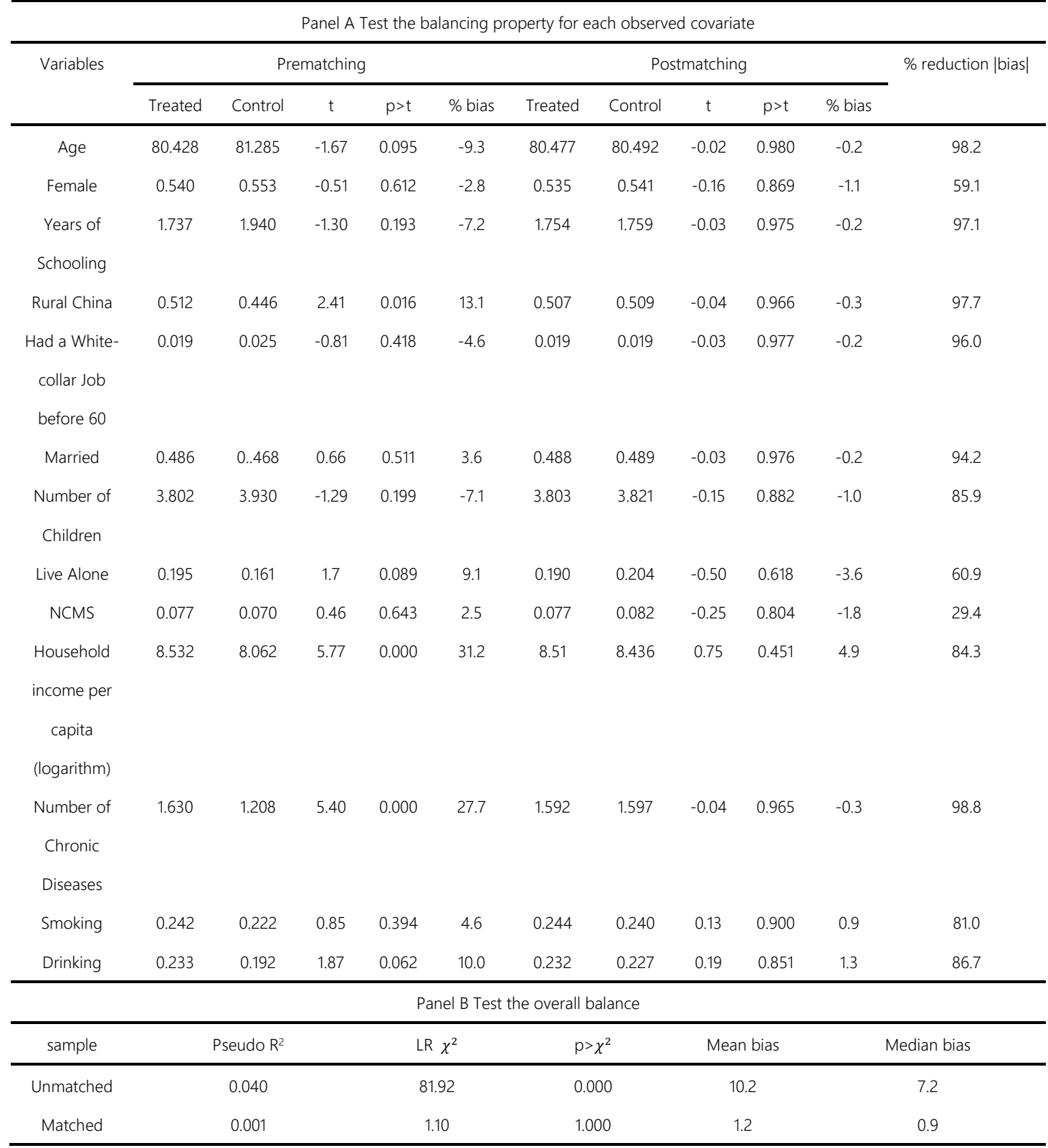



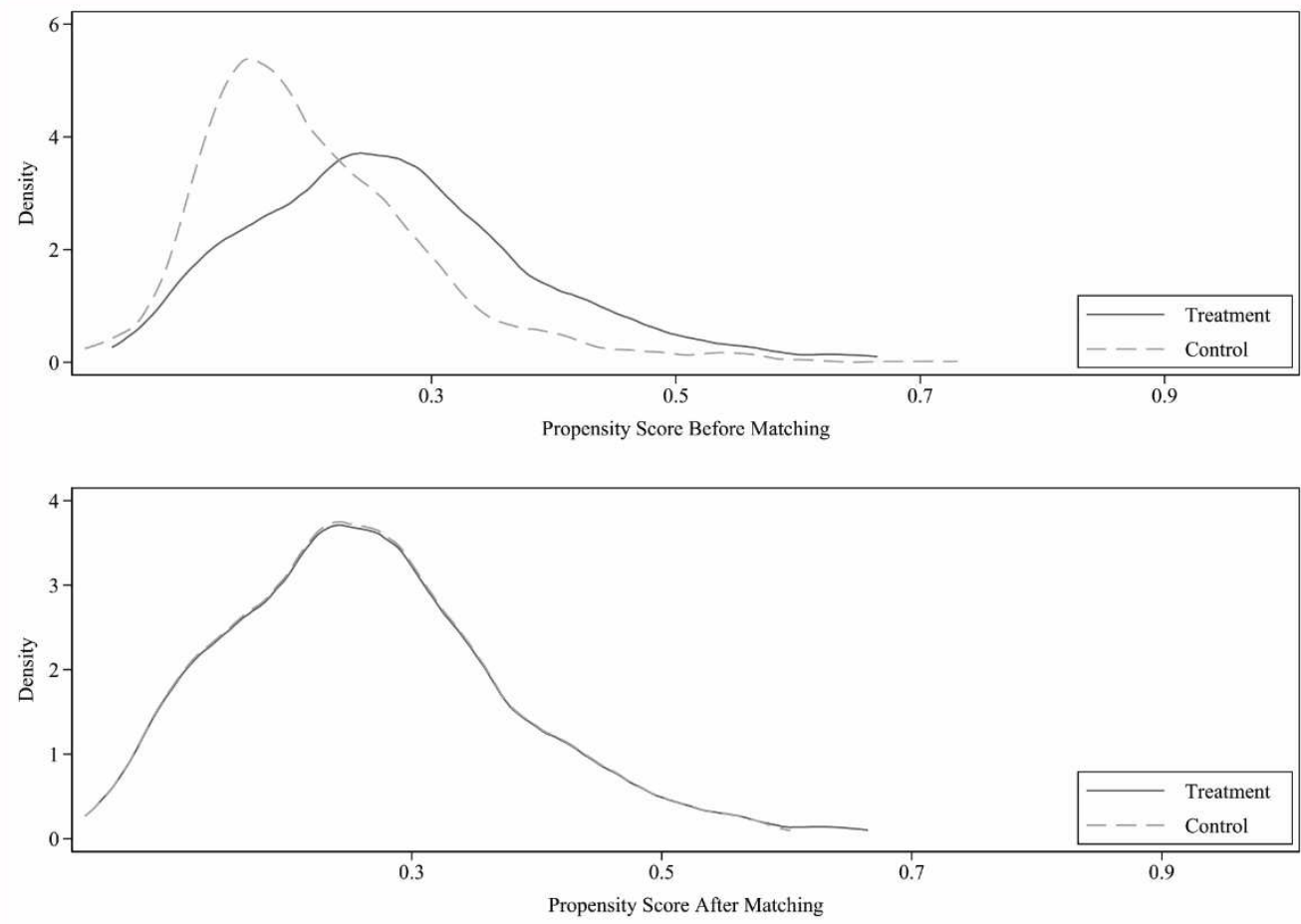

1

2 Figure A1. The distribution of density of propensity score before and after matching 